\title{
Rotator cuff repair protocols: a survey of current New Zealand practice
}

Bronwyn Harman Dip Phys, Adv Dip Phys (OMT), MHSc (Hons)

Lecturer, Department of Physiotherapy, Faculty of Health and Environmental Sciences, Auckland University of Technology

Margie Olds MHSC (Hons)

Clinical Educator, Department of Physiotherapy, Faculty of Health and Environmental Sciences, Auckland University of Technology

\section{ABSTRACT}

Rehabilitation following surgical rotator cuff repair may enable return to pre-injury function. Optimal recovery is facilitated by the use of post-operative protocols that enhance communication between the surgeon and physiotherapist. The purpose of this study was to describe the rotator cuff repair rehabilitation protocols currently used in New Zealand. Orthopaedic surgeons practising in New Zealand were contacted to determine their current rehabilitation protocols. Length of immobilisation and exercise progression following surgical repair were analysed across weekly timeframes from the day of surgery until nine months post-operatively. The response rate was 55\% with protocols received from 31 surgeons. Post-surgical rotator cuff protocols currently in use in New Zealand are predominantly determined by weekly time periods which vary from surgeon to surgeon. Immobilisation was most commonly recommended for four to six weeks, pendular exercises were started the week of surgery, active exercises commenced at six weeks, followed by resisted exercises at twelve weeks. Few protocols based the rehabilitation upon tissue quality and size of rotator cuff tear. Level of detail regarding sets and repetitions of exercises also differed between protocols. Variability across protocols was evident regarding the recommended activity level and time period from surgery. Consideration of tissue quality, size of the tear and strength of the repair is recommended to facilitate optimal results.

\section{Harman, B., Olds, M. (2017) Rotator cuff repair protocols: a survey of current New Zealand practice. New Zealand Journal of Physiotherapy 45(1): 24-30. doi: 10.15619/NZJP/45.1.04}

Key words: Surgical repair, Tendon, Rehabilitation, Rotator cuff

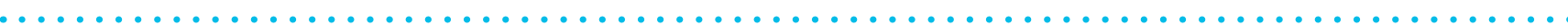

\section{INTRODUCTION}

Rotator cuff tears are a frequent source of shoulder pain, with a prevalence of $25 \%$ in people over 60 years, rising to $50 \%$ in those over the age of 80 years (Tashjian, 2012; Yamamoto et al., 2010). A recent study in the United States of America (USA) reported that the incidence of rotator cuff repairs has increased by 238\% since 1995 (Ensor, Kwon, DiBeneditto, Zuckerman, \& Rokito, 2013). This increase may be due to improved imaging techniques, increased availability of surgery and an ageing population, which may lead to further demand for surgery (Ensor et al., 2013). In New Zealand, the total number of rotator cuff repairs in the 2015/2016 financial year was 3,205, with associated surgical and physiotherapy costs borne by the Accident Compensation Corporation (ACC) of $\$ 31,125,063$ and $\$ 1,116,506$, respectively (ACC, personal communication, September 7, 2016). In the USA, between 200,000 and 300,000 rotator cuff tears are surgically repaired each year (Colvin, Harrison, Flatow, Egorova, \& Moskowitz, 2012), at a cost of between $\$ U S 10,000$ and $\$ U S 17,000$ per surgery (Vitale et al., 2007); total annual expenditure in the USA is estimated at between \$US3-12 billion dollars (McElvany, McGoldrick, Gee, Neradilek, \& Matsen, 2015). Additional post-surgical rehabilitation costs of approximately $\$ U S 3,000$ per person (Arshi et al., 2015) mean that the total expenditure is considerable.

Rehabilitation following surgical repair can involve significant periods of time, with some protocols providing guidance to therapists for 4 to 6 months, but this can be extended by complications such as tendon non-healing, which varies from 12\% to 94\% (Boileau et al., 2005; Galatz, Ball, Teefey, Middleton, \& Yamaguchi, 2004; Kim et al., 2012; Thigpen et al., 2016). Tendon healing can be affected by various surgical, mechanical and patient specific factors following surgical repair (Mall, Tanaka, Choi, \& Paletta, 2014). Patient specific factors associated with decreased healing include large sized tears $(>3 \mathrm{~cm})$, an increased number of affected tendons (Boileau et al., 2005; Cho \& Rhee, 2009; Gulotta et al., 2011; Le, Wu, Lam, \& Murrell, 2014), fatty infiltration (Cho \& Rhee, 2009; Chung, Kim, Kim, Kim, \& Oh, 2013), the extent of tendon retraction (Charousset, Bellaïche, Kalra, \& Petrover, 2010; Meyer, Wieser, Farshad, \& Gerber, 2012) and the quality of bone stock (Chung, Oh, Gong, Kim, \& Kim, 2011). Decreased tendon-to-bone healing has also been associated with increased patient age (Boileau et al., 2005; Cho \& Rhee 2009; Gulotta et al., 2011). Excessive load on the tendon in the early post-operative phase has been shown to be detrimental to healing (Sonnabend, Howlett, \& Young, 2010; Thomopoulos, Williams, \& Soslowsky, 2003). However, non-healing may also result from complete lack of load, as a balance of load is required to promote optimal healing (Galatz et al., 2009). Optimally loading the tendon has been shown to increase fibroblast proliferation, and synthesis and organisation of collagen (Liu, Yang, Al-Shaikh, \& Lane, 1995). Early loading of the tendon with passive range of motion (ROM) exercises may not be detrimental to healing in small to medium sized tears $(<3 \mathrm{~cm})$ (Keener, Galatz, Stobbs-Cucchi, Patton, \& Yamaguchi, 2014; Kim et al., 2012). However, people 
with larger sized tears $(>3 \mathrm{~cm}$ ) may be prone to an increased retear rate with early passive ROM (Lee, Cho, \& Rhee, 2012).

Rehabilitation following rotator cuff repair is guided by post-surgical protocols which may recommend a period of immobilisation followed by progressive loading of the tendon through passive, active and resisted exercise (Littlewood et al., 2015). Currently, there are a lack of detailed published guidelines for rehabilitation following rotator cuff repair in New Zealand (Accident Compensation Corporation, 2003). The aim of this study was to describe rotator cuff repair rehabilitation protocols currently used in New Zealand. It was hypothesised that, although there would be variation amongst protocols, they would contain information regarding restrictions on immobilisation, passive and active ROM, and resisted exercises.

\section{METHODS}

In order to establish the current protocols available to New Zealand physiotherapists, orthopaedic surgeons with a special interest in shoulder surgery were identified via the New Zealand Orthopaedic Association and contacted for their post-surgical rotator cuff protocols, between May and December 2014. Ethical approval was not required as this study was considered a clinical audit because it did not involve human participants and the protocols received from surgeons were de-identified. In addition, some protocols were publicly available documents. A protocol was defined as any pre-printed formal protocol, website protocol, or a protocol received via email describing the implementation of various treatment modalities at specific times in order to maximise tissue healing and minimise joint stiffness (van der Meijden et al., 2012).

Data from the protocols were extracted individually by two researchers ( $\mathrm{BH}$ and $\mathrm{MO}$ ) into a standardised data form, including the length of immobilisation, and the time at which passive, active and resisted exercises were commenced, as well as the duration of these exercises (van der Meijden et al., 2012). Consensus was reached in a single meeting by the researchers if disagreement existed over the protocols.

Pendular exercises and self-assisted shoulder flexion are common passive exercises that have been previously reported in a rehabilitation protocol (Millett, Wilcox, O'Holleran, \& Warner, 2006) and were chosen to represent the passive phase of rehabilitation. As electromyographic (EMG) activity is low with these exercises, they were considered representative of passive exercise (Dockery, Wright, \& LaStayo, 1998; Long et al., 2010; McCann, Wootten, Kadaba, \& Bigliani, 1993). Active exercise was defined as "patient-generated movement" whereas resisted exercise was defined as movement undertaken with load applied (Petty, 2014). In instances where return to work/sport, and/or alternative therapies such as aquatherapy or cryotherapy were described in a protocol, these data were also extracted as such forms of therapy have been shown to enhance patient outcomes (Brady, Redfern, Macdougal, \& Williams, 2008; Osbahr, Cawley, \& Speer, 2002). Data from protocols which reported variation according to the size of the rotator cuff tear, the quality of tissue, or other variables such as the level of pain, were also documented.

\section{RESULTS}

Of the 56 surgeons who were contacted for post-surgical protocols, 31 (55\%) responded, with protocols received from a wide geographical area throughout New Zealand. Eleven were in printed form, while 18 were written directly to the researchers in an email. One protocol referred to published literature (Millet et al., 2006) and one referred to an independent website (Moon Shoulder Group, 2008).

\section{Immobilisation}

Length of immobilisation varied from no immobilisation to twelve weeks. Twelve (39\%) protocols recommended immobilisation for 6 weeks (Figure 1), and three (10\%) specifically addressed the severity of the tear by requiring immobilisation for 4 weeks in cases of a small tear, and 6 or 8 weeks for a large tear.

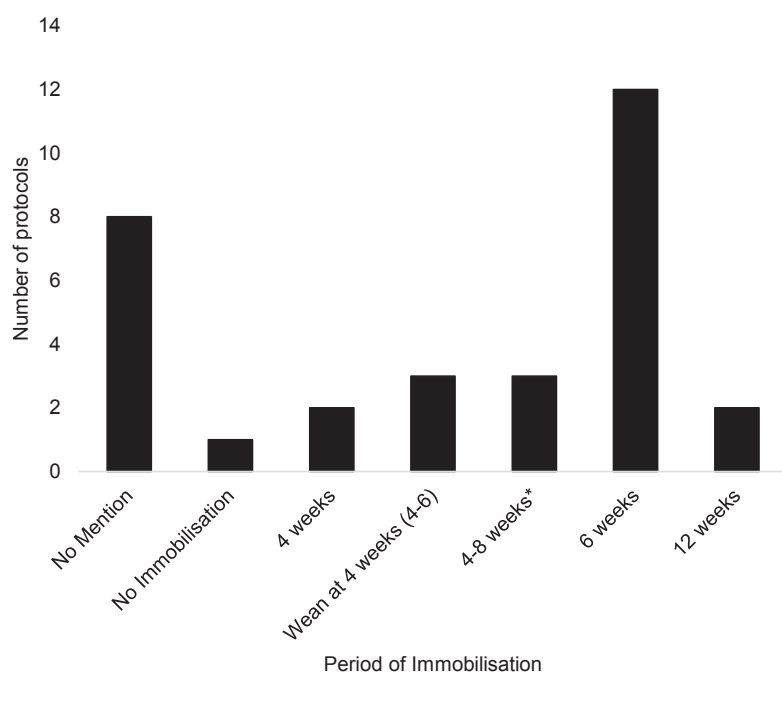

* Period of immobilisation between 4 to 8 weeks dependent on tear size

\section{Figure 1: Period of immobilisation recommended following rotator cuff repair ( $n=31$ protocols)}

\section{Passive exercise}

Pendular exercises were included in $22(71 \%)$ protocols. Eighteen of these (58\%) recommended pendular exercises in the first week following surgery (week 0) (Figure 2). One protocol introduced pendular exercises from week 1, another started these at week 2, while two delayed commencement of these exercises until week 4 . Only five (16\%) indicated the number of repetitions, which varied from two to four times per day. A third of the protocols (29\%) did not contain any information on pendular exercises. 


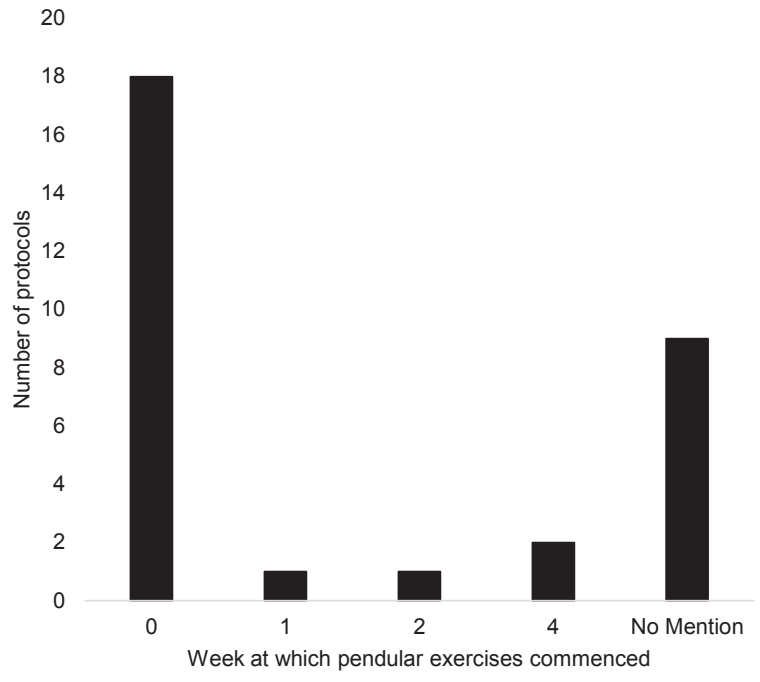

Figure 2: Time of commencement of pendular exercises ( $n=31$ protocols)

Self-assisted shoulder flexion was recommended by 26 (84\%) protocols. Two (6\%) of these recommended an alternative exercise of table slides instead of supine self-assisted shoulder flexion. Eleven (35\%) protocols commenced self-assisted shoulder flexion the week of surgery (week 0) (Figure 3). Seven $(23 \%)$ restricted the range of shoulder flexion to $90^{\circ}$ for a stipulated period of time, varying between 4 to 12 weeks. One protocol limited flexion to $45^{\circ}$ until week 3 , after which flexion could be increased to $90^{\circ}$ until week 6 . Four (13\%) protocols accounted for tissue quality and size of the tear, by varying the commencement of self-assisted flexion from week 2 to 4 (two protocols) or from week 4 to 6 (two protocols). The two protocols $(6 \%)$ that recommended table slides commenced this exercise at either week 0 , or week 4 . Five (16\%) protocols made no mention of any passive flexion exercises.

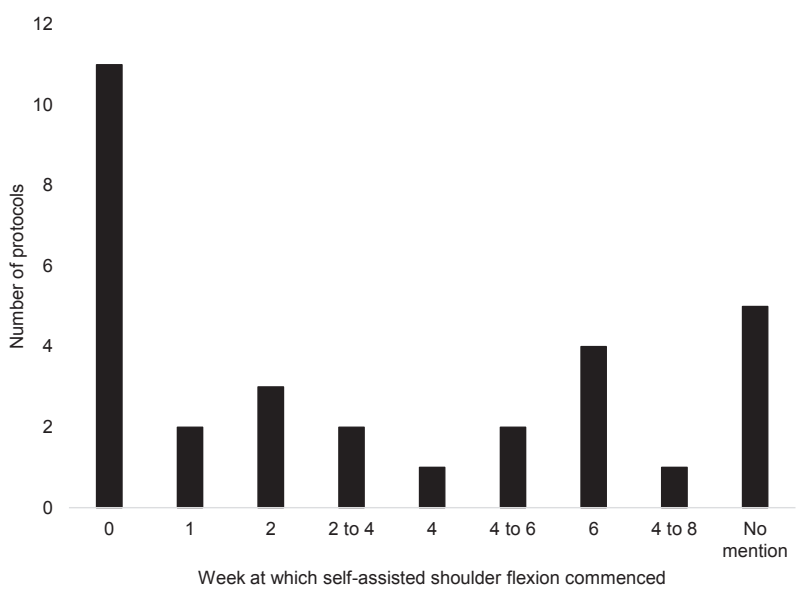

Figure 3: Time of commencement of self-assisted shoulder flexion ( $n=31$ protocols)

Active exercise

All 31 protocols recommended the use of active exercise (Figure 4), commencing with exercises such as active shoulder flexion and light weight waist level activities. Active exercise commenced at week 6 in twelve (39\%) protocols, at week 8 in six $(19 \%)$, and at week 12 in five (16\%) protocols. Six (19\%) recommended adjusting the commencement of active exercise dependent upon other variables (e.g. size of tear and quality of tissue) at 4 to 6 weeks, 4 to 8 weeks, or 8 to 12 weeks. Active ROM was restricted until full passive ROM was achieved in one protocol.

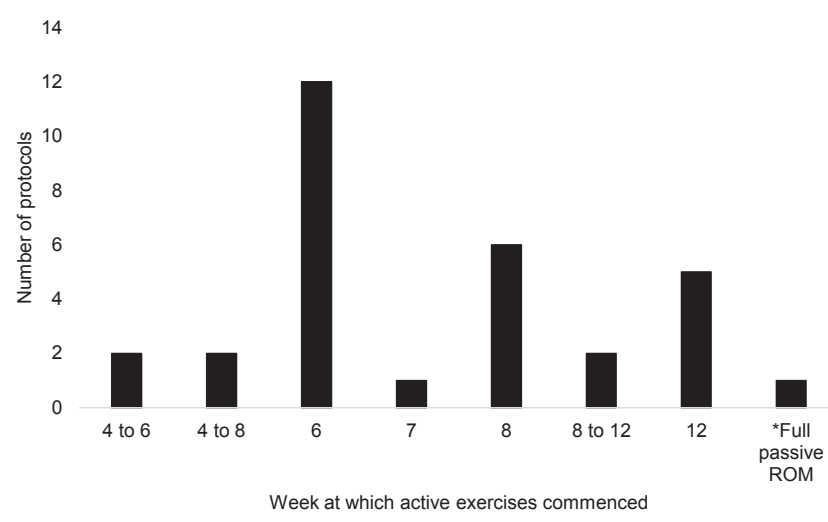

* Active movement commenced when full passive ROM was gained

Figure 4: Time of commencement of active exercises $(n=31$ protocols)

\section{Resisted exercise}

All 31 (100\%) protocols recommended resisted exercise during rehabilitation (Figure 5). Sixteen (52\%) protocols recommended commencement of resisted exercise at 12 weeks following surgery. Some protocols advised the physiotherapist to delay the commencement of resisted exercises depending on tear size and tissue quality. One (3\%) protocol advised commencement from 8 to 12 weeks, two (6\%) advised from 8 to 16 weeks and three $(10 \%)$ advised from 10 to 12 weeks. Five (16\%) protocols recommended starting resisted exercises with isometric exercises. Only two (6\%) protocols indicated the number of sets and repetitions for strengthening. One recommended resisted dumb-bell exercises three times per week with three to four sets of 10 repetitions. The other recommended three sets of 10 repetitions of Thera-Band $\AA$ (The Hygenic Corporation, Akron, Ohio) exercises, three times per day.

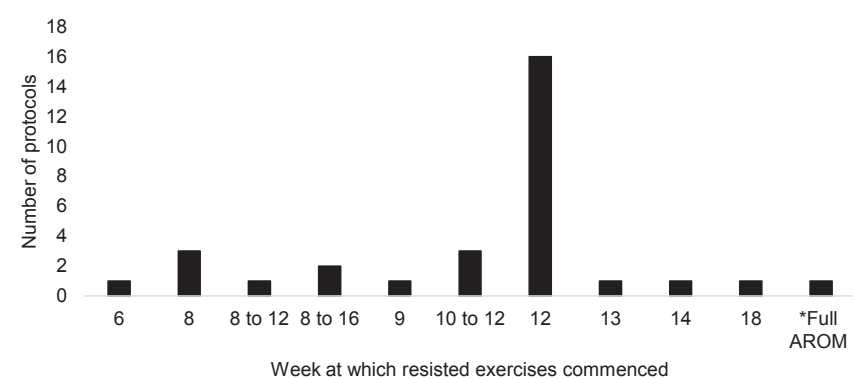

${ }^{*}$ Resisted exercises were commenced when full AROM was gained

Figure 5: Time of commencement of resisted exercises ( $n=31$ protocols) 


\section{Return to work or sport}

Time frames for return to work were recommended by three $(10 \%)$ protocols. Two (6\%) suggested return to light work at 3 to 4 months, and heavy work at 6 to 9 months after surgery. One (3\%) recommended return to work at 6 months. Return to sport was recommended in four (13\%) protocols at various time frames, from 4 to 9 months post-operatively.

\section{Other variables related to rehabilitation}

Rehabilitation was altered depending on variables including the size of the tear, quality of the tissue, quality of surgical fixation, presence of pain and whether aquatherapy or cryotherapy was added. The size of the rotator cuff tear was considered in twelve (39\%) protocols with progression delayed for large sized tears (Figure 6). Delayed progression of rehabilitation was recommended in seven (23\%) protocols when the tissue was of poor quality and in five (16\%) when tendon fixation was considered poor. Ten (32\%) altered the rehabilitation according to the particular tendon involved, and treatment was varied in $10(32 \%)$ if pain was present. Seven recommended slowing progression to reduce pain, while one advised caution with rapid progression if the patient had minimal pain. Two (6\%) documented that ROM exercises be undertaken to the point of pain. Aquatic therapy was proposed as an adjunct in three $(10 \%)$ protocols, while seven $(23 \%)$ incorporated cryotherapy into the rehabilitation programme.

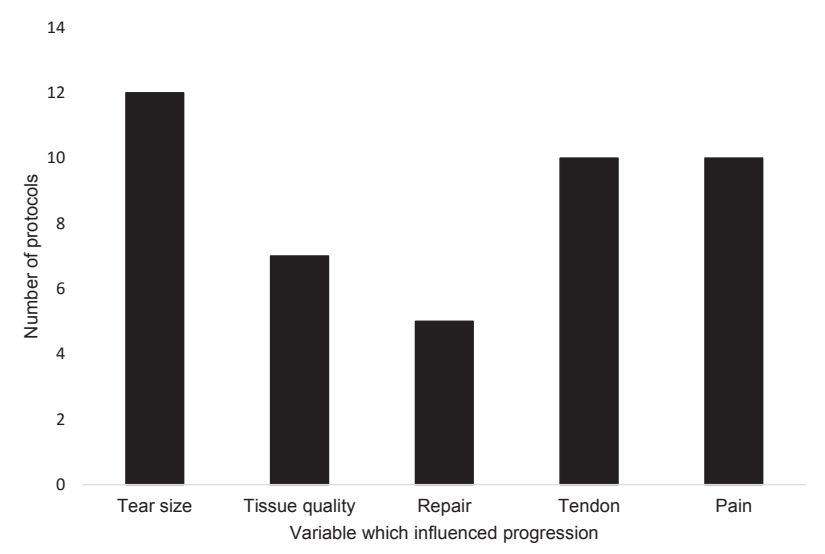

* Some protocols included more than one variable

Figure 6: Other variables considered to influence rehabilitation of rotator cuff repairs ( $n=31$ protocols)

\section{DISCUSSION}

This study established that a wide variety of rotator cuff post-operative protocols are currently in use throughout New Zealand and highlights the need for further discussion regarding the optimal management following rotator cuff repair. The majority of protocols divided the gradation of rehabilitation into phases, including immobilisation, passive, active and resisted exercises; however, considerable variation was evident in the commencement and definition of these phases. Few protocols considered individual factors such as size of the tear, tissue quality or strength of the fixation.

\section{Immobilisation}

Immobilisation following a rotator cuff repair has been shown to increase viscoelastic properties and collagen organisation of the repaired tendon (Parsons et al., 2010; Thomopoulos et al., 2003). In the current study, the length of immobilisation varied across the protocols from no immobilisation to 12 weeks. The majority of protocols recommended immobilisation for 4 to 6 weeks, which is similar to current practice in the United Kingdom (Littlewood \& Bateman, 2015). A review of randomised controlled trials, which compared the effect of early versus delayed rehabilitation on outcomes and re-tear rates, showed no adverse effect on outcomes or re-tear rates with early rehabilitation (Littlewood et al., 2015). Some of these studies may have been underpowered however, potentially leading to a Type II error or a false negative result (Littlewood et al., 2015). An optimal balance may be required between some immobilisation to protect the repair and some load to promote healing within the tendon (Galatz et al., 2009). The consensus statement from the American Society of Shoulder and Elbow Therapists (ASSET) on rehabilitation following arthroscopic rotator cuff repair recommends immobilisation for a period of four to six weeks (Thigpen at al., 2016). Both the ASSET consensus statement (Thigpen et al., 2016) and a recent sytematic review (Thomson, Jukes \& Lewis, 2016) suggest consideration of tear size and tissue quality to be important, with the latter also advocating a period of immobilisation for four to six weeks for a large tear.

\section{Passive exercise}

The current study found that in general pendular exercises were initiated up to 4 weeks post-operatively. Small pendular exercises $(20 \mathrm{~cm}$ in diameter) performed in a position of trunk flexion generate less than $10 \%$ maximal voluntary contraction (MVC) of supraspinatus, whereas large, incorrectly performed pendular exercises can generate more than 15\% of MVC of supraspinatus (Long et al., 2010). The ASSET consensus statement recommends protected passive ROM during the first 6 weeks after rotator cuff repair of small to medium sized tears (< $4 \mathrm{~cm}$ ), with EMG activity of $15 \%$ (Thigpen et al., 2016). Previous studies have defined passive exercise as below 20\% MVC (Ellsworth, Mullaney, Nicholas, McHugh, \& Tyler, 2004; Smith et al., 2006), below 10\% MVC (Uhl, Muir, \& Lawson, 2010), or below 5\% MVC (Dockery et al., 1998).

The use of self-assisted shoulder flexion was initiated between 0-8 weeks post-operatively. As demonstrated in EMG studies, self-assisted shoulder flexion involves less supraspinatus activity than reciprocal pulleys; however, it has a higher level of muscle activity than pendular exercises (Dockery et al., 1998; Gurney et al., 2016; Murphy, McDermott, Petersen, Johnson, \& Baxter, 2013). Many protocols did not identify the specific passive exercises to be used in rehabilitation yet therapists should be aware of the variability in EMG activity across passive exercises for individual muscles (Gurney et al., 2016).

\section{Active exercise}

Some of the protocols (39\%) reported commencement of active exercise at 6 weeks following rotator cuff repair, but this is slightly later than current practice in the United Kingdom where active exercises commence between 4 to 6 weeks (Littlewood \& Bateman, 2015). The strength of the repaired tendon is likely to only be up to $30 \%$ of normal at 6 weeks post-operatively and therefore unlikely to be able to withstand large loads or repetitive loading (Gerber, Schneeberger, Perren, \& Nyffeler, 
1999; Thigpen et al., 2016). Removal of an immobilisation sling necessitates active movement (Gurney et al., 2016). Few protocols provided examples of specific active exercises. The ASSET consensus statement recommends light muscle activities at chest level or below (Thigpen et al., 2016).

\section{Resisted exercise}

Over 50\% (16/31) of protocols implemented resisted exercises at 12 weeks post-operatively. This is in agreement with a study on primates which found an increased number of Sharpey fibres connecting tendon to bone at 12 weeks following rotator cuff repair (Sonnabend et al., 2010). In animal studies, the strength of the repair is between $29 \%$ and $50 \%$ at 12 weeks (Gerber et al., 1999; Thigpen et al., 2016). The consensus statement by ASSET recommends progressing to strengthening from 12 weeks and to start with light strengthening when pain is well controlled ( $<2 / 10$ on a numeric pain rating scale) and sufficient passive ROM has been achieved (Thigpen et al., 2016). Some protocols started a resistance programme with isometric exercises; however, care is needed as maximal isometric exercise may load the tendon more than isotonic exercise (Thigpen et al., 2016). Pain free exercise at low levels of MVC can provide a mechanical stimulus for mechanotransduction (Khan \& Scott, 2009) and the progressive loading of tissue results in collagen deposition and increased tensile strength of the tendon (Lin, Cardenas, \& Soslowsky, 2004). While few protocols indicated the number of sets and repetitions for strengthening, the two protocols that did provide specific guidelines varied from performing resisted exercises three times per week, to three times per day. As improvement in strength is dependent on the type of exercise intensity and frequency prescribed (Kraemer \& Ratamess, 2004), further detail regarding the volume of resisted exercises may enhance patient outcome. Additionally, the volume of load should be individualised dependent on patient goals, and other variables such as the quality of tissue.

\section{Cryotherapy and aquatic therapy}

Seven $(23 \%)$ protocols recommended the use of cryotherapy. The benefit of pain relief from cryotherapy has been demonstrated post-operatively following shoulder surgery (Osbahr et al., 2002; Singh, Osbahr, Holovacs, Cawley, \& Speer, 2001; Speer, Warren, \& Horowitz, 1996). However no difference in pain relief has been found between a commercial compressive cryotherapy device compared to a standard ice wrap (Kraeutler, Reynolds, Long, \& McCarty, 2015). Only three $(10 \%)$ protocols included aquatic therapy, which has been shown to increase ROM following rotator cuff repair (Brady et al., 2008). Aquatic therapy also produces less muscle activity with shoulder elevation when performed slowly compared to dry land exercise (Kelly, Roskin, Kirkendall, \& Speer, 2000). Slow speed aquatherapy for ROM is recommended from 3 to 6 weeks by ASSET and cryotherapy is recommended for pain relief from the first day post-operatively (Thigpen et al., 2016).

\section{Other variables}

Less than $40 \%$ of the protocols considered other factors such as tear size, tissue quality, strength of the fixation or the tendon involved and yet rehabilitation based on these variables has been advocated (Killian \& Cavanaugh, 2014; Pabian, Rothschild, \& Schwartzberg, 2011; van der Meijden et al., 2012; Thigpen et al., 2016; Thomson et al., 2016). A recent meta-analysis reported that increased re-tear rates were associated with larger tear size, advanced age, and more fatty infiltration (McElvany et al., 2015). Few of the protocols left the progression of rehabilitation to the discretion of the therapist, and none reported informing the therapist of the tear size, type of repair, or degree of fatty infiltration. Given that these variables are significantly associated with re-tear rates following rotator cuff repair, communication regarding these variables may allow therapists to optimise individual progression to achieve better outcomes.

Ten (32\%) protocols considered pain during rehabilitation with most of these suggesting adjustment (slowing down) in cases of excessive pain. Only one protocol suggested that minimal pain may be detrimental, with the temptation for the patient to progress too quickly and risk re-tear. Patients who have a rapid early improvement in clinical outcome and/or are less compliant with their rehabilitation are at greater risk of re-tear (Ahmad, Haber, \& Bokor, 2015). Further research into individualised rehabilitation programmes, based upon such factors as size of tear, location, quality of fixation, tissue quality, and general health is warranted (Boileau et al., 2005; Killian \& Cavanaugh, 2014; Murphy et al., 2013; Thigpen et al., 2016; Thomson et al., 2016; van der Meijden et al., 2012).

\section{Study limitations}

A limitation of this study was the $55 \%$ response rate from the surgeons who were approached to participate. While this study replicated the information currently available to New Zealand therapists by extracting data from each protocol, the use of a standardised questionnaire completed by participating surgeons may have provided more detail regarding surgical protocols (e.g. sets and repetitions of exercises). In addition, collecting data from different modes such as email, printed protocols and website information may have resulted in a variation in the level of detail provided. Future research could also investigate therapist adherence to protocol implementation or therapist interpretation of the information contained in protocols.

\section{CONCLUSION}

Variability is evident amongst the protocols currently in use in New Zealand, but some similarities across protocols alongside comparisons with international studies can be used to guide therapeutic rehabilitation following rotator cuff repair. The majority of protocols recommend immobilisation of the shoulder following a repair for 4 to 6 weeks. Passive exercises (or exercises that produce low-levels of muscle activity as determined with EMG) commence between 0-8 weeks and most protocols progress to active exercises between 6 and 8 weeks. The majority of protocols recommend starting resisted exercises at 12 weeks which is when the tendon may be sufficiently healed to start exercise against resistance. Communication from the surgeon to the therapist regarding tear size, tissue quality and strength of fixation may reduce the risk of re-tear following surgery. Further investigation into the optimisation of rehabilitation based upon individual patient factors may result in more timely return to full function, following a rotator cuff repair. 


\section{KEY POINTS}

1. This study adds to current knowledge about rotator cuff repair protocols by identifying the variability across protocols currently in use in New Zealand.

2. Most protocols recommended immobilisation for four to six weeks, followed by passive and active exercises, with commencement of resisted strengthening at twelve weeks.

3. Identification and communication of variables which impact on rehabilitation following surgical repair, such as tear size, degree of fatty infiltration, and type of repair, could enhance outcomes for individuals following rotator cuff repair.

4. Future research could investigate the benefit of an individually tailored rehabilitation programme.

\section{DISCLOSURES}

No conflicts of interest have been identified for this research.

The authors would like to acknowledge the co-operation of New Zealand orthopaedic surgeons in providing their postoperative rehabilitation protocols.

\section{CORRESPONDING AUTHOR:}

Bronwyn Harman

Department of Physiotherapy, Faculty of Health and Environmental Sciences, Auckland University of Technology, Private Bag 92006, Auckland 1142, New Zealand. Telephone: +64 99219999 ext 7518. Email: bronwyn.harman@aut.ac.nz

\section{REFERENCES}

Accident Compensation Corporation. (2003). The diagnosis and management of soft tissue shoulder injuries and related disorders. Retrieved 11 August, 2016, from www.acc.co.nz/PRD_EXT_CSMP/groups/ external_communications/documents/guide/wcm001684.pdf.

Ahmad, S., Haber, M., \& Bokor, D. J. (2015). The influence of intraoperative factors and postoperative rehabilitation compliance on the integrity of the rotator cuff after arthroscopic repair. Journal of Shoulder and Elbow Surgery, 24(2), 229-235. doi:10.1016/j.jse.2014.06.050.

Arshi, A., Kabir, N., Cohen, J. R., Lord, E. L., Wang, J. C., McAllister, D. R., \& Petrigliano, F. A. (2015). Utilization and costs of postoperative physical therapy after rotator cuff repair: A comparison of privately insured and medicare patients. Arthroscopy, 31(12), 2392-2399.e2391. doi:10.1016/j. arthro.2015.06.018.

Boileau, P., Brassart, N., Watkinson, D. J., Carles, M., Hatzidakis, A. M., \& Krishnan, S. G. (2005). Arthroscopic repair of full-thickness tears of the supraspinatus: Does the tendon really heal? Journal of Bone and Joint Surgery - American Volume, 87(6), 1229-1240. doi:10.2106/jbjs.d.02035.

Brady, B., Redfern, J., Macdougal, G., \& Williams, J. (2008). The addition of aquatic therapy to rehabilitation following surgical rotator cuff repair: $A$ feasibility study. Physiotherapy Research International, 13(3), 153-161. doi:10.1002/pri.403

Charousset, C., Bellaïche, L., Kalra, K., \& Petrover, D. (2010). Arthroscopic repair of full-thickness rotator cuff tears: Is there tendon healing in patients aged 65 years or older? Arthroscopy - Journal of Arthroscopic and Related Surgery, 26(3), 302-309. doi:10.1016/j.arthro.2009.08.027.

Cho, N. S., \& Rhee, Y. G. (2009). The factors affecting the clinical outcome and integrity of arthroscopically repaired rotator cuff tears of the shoulder. Clinics in Orthopedic Surgery, 1(2), 96-104. doi:10.4055/cios.2009.1.2.96.
Chung, S. W., Kim, J. Y., Kim, M. H., Kim, S. H., \& Oh, J. H. (2013). Arthroscopic repair of massive rotator cuff tears: Outcome and analysis of factors associated with healing failure or poor postoperative function. American Journal of Sports Medicine, 41(7), 1674-1683. doi:10.1177/0363546513485719.

Chung, S. W., Oh, J. H., Gong, H. S., Kim, J. Y., \& Kim, S. H. (2011). Factors affecting rotator cuff healing after arthroscopic repair: Osteoporosis as one of the independent risk factors. American Journal of Sports Medicine, 39(10), 2099-2107. doi:10.1177/0363546511415659.

Colvin, A. C., Harrison, A. K., Flatow, E. L., Egorova, N., \& Moskowitz, A. (2012). National trends in rotator cuff repair. Journal of Bone and Joint Surgery - Series A, 94(3), 227-233. doi:10.2106/jbjs.j.00739.

Dockery, M. L., Wright, T. W., \& LaStayo, P. C. (1998). Electromyography of the shoulder: An analysis of passive modes of exercise. Orthopedics, 21(11), 1181-1184

Ellsworth, A. A., Mullaney, M. J., Nicholas, S. J., McHugh, M. P., \& Tyler, T. F. (2004). Electromyography of selected shoulder musculature during unweighted and weighted pendulum exercises. Journal of Orthopaedic and Sports Physical Therapy, 34(1), A57-A57.

Ensor, K. L., Kwon, Y. W., DiBeneditto, M. R., Zuckerman, J. D., \& Rokito, A S. (2013). The rising incidence of rotator cuff repairs. Journal of Shoulder and Elbow Surgery, 22(12), 1628-1632. doi:10.1016/j.jse.2013.01.006.

Galatz, L. M., Ball, C. M., Teefey, S. A., Middleton, W. D., \& Yamaguchi, K. (2004). The outcome and repair integrity of completely arthroscopically repaired large and massive rotator cuff tears. Journal of Bone and Joint Surgery - American Volume, 86(2), 219-224.

Galatz, L. M., Charlton, N., Das, R., Kim, H. M., Havlioglu, N., \& Thomopoulos, S. (2009). Complete removal of load is detrimental to rotator cuff healing. Journal of Shoulder and Elbow Surgery, 18(5), 669675. doi:10.1016/j.jse.2009.02.016

Gerber, C., Schneeberger, A. G., Perren, S. M., \& Nyffeler, R. W. (1999). Experimental rotator cuff repair. A preliminary study. Journal of Bone and Joint Surgery - American Volume, 81(9), 1281-1290

Gulotta, L. V., Nho, S. J., Dodson, C. C., Adler, R. S., Altchek, D. W., \& MacGillivray, J. D. (2011). Prospective evaluation of arthroscopic rotator cuff repairs at 5 years: Part II-prognostic factors for clinical and radiographic outcomes. Journal of Shoulder and Elbow Surgery, 20(6), 941-946. doi:10.1016/j.jse.2011.03.028.

Gurney, A. B., Mermier, C., Laplante, M., Majumdar, A., O'Neill, K., Shewman, T., \& Gurney, J. G. (2016). Shoulder electromyography measurements during activities of daily living and routine rehabilitation exercises. Journal of Orthopaedic and Sports Physical Therapy, 46(5), 375383 379p. doi:10.2519/jospt.2016.6090.

Keener, J. D., Galatz, L. M., Stobbs-Cucchi, G., Patton, R., \& Yamaguchi, K. (2014). Rehabilitation following arthroscopic rotator cuff repair : A prospective randomized trial of immobilization compared with early motion. Journal of Bone and Joint Surgery - American Volume, 96(1), 11 19. doi:10.2106/jbjs.m.00034.

Kelly, B. T., Roskin, L. A., Kirkendall, D. T., \& Speer, K. P. (2000). Shoulder muscle activation during aquatic and dry land exercises in nonimpaired subjects. Journal of Orthopaedic and Sports Physical Therapy, 30(4), 204210.

Khan, K. M., \& Scott, A. (2009). Mechanotherapy: How physical therapists' prescription of exercise promotes tissue repair. British Journal of Sports Medicine, 43(4), 247-252. doi:10.1136/bjsm.2008.054239.

Killian, S. E., \& Cavanaugh, J. T. (2014). Rehabilitation guidelines after rotator cuff repair. Techniques in Shoulder and Elbow Surgery, 15(1), 8-12. doi: doi:10.1097/bte.0000000000000017

Kim, Y. S., Chung, S. W., Kim, J. Y., Ok, J. H., Park, I., \& Oh, J. H. (2012). Is early passive motion exercise necessary after arthroscopic rotator cuff repair? American Journal of Sports Medicine, 40(4), 815-821. doi:10.1177/0363546511434287 
Kraemer, W. J., \& Ratamess, N. A. (2004). Fundamentals of resistance training: Progression and exercise prescription. Medicine and Science in Sports and Exercise, 36(4), 674-688. doi:10.1249/01. mss.0000121945.36635.61.

Kraeutler, M. J., Reynolds, K. A., Long, C., \& McCarty, E. C. (2015). Shoulder: Compressive cryotherapy versus ice-a prospective, randomized study on postoperative pain in patients undergoing arthroscopic rotator cuff repair or subacromial decompression. Journal of Shoulder and Elbow Surgery, 24, 854-859. doi:10.1016/j.jse.2015.02.004.

Le, B. T. N., Wu, X. L., Lam, P. H., \& Murrell, G. A. C. (2014). Factors predicting rotator cuff retears: An analysis of 1000 consecutive rotator cuff repairs. American Journal of Sports Medicine, 42(5), 1134-1142. doi:10.1177/0363546514525336.

Lee, B. G., Cho, N. S., \& Rhee, Y. G. (2012). Effect of two rehabilitation protocols on range of motion and healing rates after arthroscopic rotator cuff repair: Aggressive versus limited early passive exercises. Arthroscopy, 28(1), 34-42. doi:10.1016/j.arthro.2011.07.012.

Lin, T. W., Cardenas, L., \& Soslowsky, L. J. (2004). Biomechanics of tendon injury and repair. Journal of Biomechanics, 37(6), 865-877. doi:10.1016/j. jbiomech.2003.11.005.

Littlewood, C., \& Bateman, M. (2015). Rehabilitation following rotator cuff repair: A survey of current UK practice. Shoulder and Elbow, 7(3), 193204. doi:10.1177/1758573215571679.

Littlewood, C., Bateman, M., Clark, D., Selfe, J., Watkinson, D., Walton, M., \& Funk, L. (2015). Rehabilitation following rotator cuff repair: A systematic review. Shoulder and Elbow, 7(2), 115-124. doi:10.1177/1758573214567702.

Liu, S. H., Yang, R.-S., Al-Shaikh, R., \& Lane, J. M. (1995). Collagen in tendon, ligament, and bone healing: A current review. Clinical Orthopaedics and Related Research, 318, 265-278.

Long, J. L., Ruberte Thiele, R. A., Skendzel, J. G., Jeon, J., Hughes, R. E., Miller, B. S., \& Carpenter, J. E. (2010). Activation of the shoulder musculature during pendulum exercises and light activities. Journal of Orthopaedic and Sports Physical Therapy, 40(4), 230-237. doi:10.2519/ jospt.2010.3095.

Mall, N. A., Tanaka, M. J., Choi, L. S., \& Paletta Jr, G. A. (2014). Factors affecting rotator cuff healing. Journal of Bone and Joint Surgery American Volume, 96(9), 778-788. doi:10.2106/jbjs.m.00583.

McCann, P. D., Wootten, M. E., Kadaba, M. P., \& Bigliani, L. U. (1993). A kinematic and electromyographic study of shoulder rehabilitation exercises. Clinical Orthopaedics and Related Research (288), 179-188.

McElvany, M. D., McGoldrick, E., Gee, A. O., Neradilek, M. B., \& Matsen, F. A. (2015). Rotator cuff repair: Published evidence on factors associated with repair integrity and clinical outcome. American Journal of Sports Medicine, 43(2), 491-500. doi:10.1177/0363546514529644.

Meyer, D. C., Wieser, K., Farshad, M., \& Gerber, C. (2012). Retraction of supraspinatus muscle and tendon as predictors of success of rotator cuff repair. American Journal of Sports Medicine, 40(10), 2242-2247. doi:10.1177/0363546512457587.

Millett, P. J., Wilcox, R. B., O'Holleran, J. D., \& Warner, J. J. P. (2006). Rehabilitation of the rotator cuff: An evaluation-based approach. Journal of the American Academy of Orthopaedic Surgeons, 14(11), 599-609.

Moon Shoulder Group. (2008). Rotator cuff rehabilitation therapist directed program. Retrieved from http://moonshoulder.com/booklets/Therapist\%20 Directed\%20Cuff\%20Rehab\%208.12.pdf.

Murphy, C. A., McDermott, W. J., Petersen, R. K., Johnson, S. E., \& Baxter, S. A. (2013). Electromyographic analysis of the rotator cuff in postoperative shoulder patients during passive rehabilitation exercises. Journal of Shoulder and Elbow Surgery, 22(1), 102-107. doi:10.1016/j. jse.2012.01.021.
Osbahr, D. C., Cawley, P. W., \& Speer, K. P. (2002). The effect of continuous cryotherapy on glenohumeral joint and subacromial space temperatures in the postoperative shoulder. Arthroscopy, 18(7), 748-754. doi:10.1053/ jars.2002.32835.

Pabian, P., Rothschild, C., \& Schwartzberg, R. (2011). Rotator cuff repair: considerations of surgical characteristics and evidence based interventions for improving muscle performance. Physical Therapy Reviews, 16(5), 374387. doi:10.1179/1743288x11y.0000000044.

Parsons, B. O., Gruson, K. I., Chen, D. D., Harrison, A. K., Gladstone, J., \& Flatow, E. L. (2010). Does slower rehabilitation after arthroscopic rotator cuff repair lead to long-term stiffness? Journal of Shoulder and Elbow Surgery, 19(7), 1034-1039. doi:10.1016/j.jse.2010.04.006.

Petty, N. J. (2014). Neuromusculoskeletal examination and assessment (4th ed.). Edinburgh: Elsevier Chirchill Livingstone.

Singh, H., Osbahr, D. C., Holovacs, T. F., Cawley, P. W., \& Speer, K. P. (2001). The efficacy of continuous cryotherapy on the postoperative shoulder: A prospective, randomized investigation. Journal of Shoulder and Elbow Surgery, 10(6), 522-525. doi:10.1067/mse.2001.118415

Smith, J., Dahm, D. L., Kaufman, K. R., Boon, A. J., Laskowski, E. R., Kotajarvi, B. R., \& Jacofsky, D. J. (2006). Electromyographic activity in the immobilized shoulder girdle musculature during scapulothoracic exercises Archives of Physical Medicine and Rehabilitation, 87(7), 923-927.

Sonnabend, D. H., Howlett, C. R., \& Young, A. A. (2010). Histological evaluation of repair of the rotator cuff in a primate model. Journal of Bone and Joint Surgery - Series B, 92(4), 586-594. doi:10.1302/0301620X.92B4.

Speer, K. P., Warren, R. F., \& Horowitz, L. (1996). The efficacy of cryotherapy in the postoperative shoulder. Journal of Shoulder and Elbow Surgery, $5(1), 62-68$

Tashjian, R. Z. (2012). Epidemiology, natural history, and indications for treatment of rotator cuff tears. Clinics in Sports Medicine, 31(4), 589-604. doi: 10.1016/j.csm.2012.07.001.

Thigpen, C. A., Shaffer, M. A., Gaunt, B. W., Leggin, B. G., Williams, G. R., \& Wilcox, R. B. (2016). The American Society of Shoulder and Elbow Therapists' consensus statement on rehabilitation following arthroscopic rotator cuff repair. Journal of Shoulder and Elbow Surgery, 25(4), 521-535. doi:10.1016/j.jse.2015.12.018.

Thomopoulos, S., Williams, G. R., \& Soslowsky, L. J. (2003). Tendon to bone healing: Differences in biomechanical, structural, and compositional properties due to a range of activity levels. Journal of Biomechanical Engineering, 125(1), 106-113. doi:10.1115/1.1536660.

Thomson, S., Jukes, C., \& Lewis, J. (2016). Rehabilitation following surgical repair of the rotator cuff: A systematic review. Physiotherapy (United Kingdom), 102(1), 20-28. doi:10.1016/j.physio.2015.08.003.

Uhl, T. L., Muir, T. A., \& Lawson, L. (2010). Electromyographical assessment of passive, active assistive, and active shoulder rehabilitation exercises. Physical Medicine and Rehabilitation Journal, 2(2), 132-141. doi:10.1016/j. pmrj.2010.01.002.

van der Meijden, O. A., Westgard, P., Chandler, Z., Gaskill, T. R., Kokmeyer, D., \& Millett, P. J. (2012). Rehabilitation after arthroscopic rotator cuff repair: Current concepts review and evidence-based guidelines. International Journal of Sports Physical Therapy, 7(2), 197-218

Vitale, M. A., Vitale, M. G., Zivin, J. G., Braman, J. P., Bigliani, L. U., \& Flatow, E. L. (2007). Rotator cuff repair: An analysis of utility scores and costeffectiveness [Article]. Journal of Shoulder and Elbow Surgery, 16(2), 181187. doi:10.1016/j.jse.2006.06.013.

Yamamoto, A., Takagishi, K., Osawa, T., Yanagawa, T., Nakajima, D., Shitara, H., \& Kobayashi, T. (2010). Prevalence and risk factors of a rotator cuff tear in the general population. Journal of Shoulder and Elbow Surgery, 19(1), 116-120. doi:10.1016/j.jse.2009.04.006. 